\title{
Module-based functional pathway enrichment analysis of a protein-protein interaction network to study the effects of intestinal microbiota depletion in mice
}

\author{
ZHEN-YI JIA, YANG XIA, DANIAN TONG, JING YAO, HONG-QI CHEN and JUN YANG \\ Department of General Surgery, Shanghai Jiao Tong University Affiliated Sixth People's Hospital, \\ Shanghai 200233, P.R. China
}

Received October 16, 2013; Accepted February 25, 2014

DOI: $10.3892 / \mathrm{mmr} .2014 .2137$

\begin{abstract}
Complex communities of microorganisms play important roles in human health, and alterations in the intestinal microbiota may induce intestinal inflammation and numerous diseases. The purpose of this study was to identify the key genes and processes affected by depletion of the intestinal microbiota in a murine model. The Affymetrix microarray dataset GSE22648 was downloaded from the Gene Expression Omnibus database, and differentially expressed genes (DEGs) were identified using the limma package in R. A protein-protein interaction (PPI) network was constructed for the DEGs using the Cytoscape software, and the network was divided into several modules using the MCODE plugin. Furthermore, the modules were functionally annotated using the PiNGO plugin, and DEG-related pathways were retrieved and analyzed using the GenMAPP software. A total of 53 DEGs were identified, of which 26 were upregulated and 27 were downregulated. The PPI network of these DEGs comprised 3 modules. The most significant module-related DEGs were the cytochrome P450 (CYP) 4B1 isozyme gene $(C Y P 4 B 1)$ in module $1, C Y P 4 F 14$ in module 2 and the tachykinin precursor 1 gene $(T A C 1)$ in module 3 . The majority of enriched pathways of module 1 and 2 were oxidation reduction pathways (metabolism of xenobiotics by CYPs) and lipid metabolism-related pathways, including linoleic acid and arachidonic acid metabolism. The neuropeptide signaling pathway was the most significantly enriched functional pathway of module 3 . In conclusion, our findings strongly suggest that intestinal microbiota depletion affects cellular metabolism and oxidation reduction pathways. In addition, this is the first time, to the best of our knowledge, that the
\end{abstract}

Correspondence to: Dr Jun Yang, Department of General Surgery, Shanghai Jiao Tong University Affiliated Sixth People's Hospital, 600 Yishan Road, Shanghai 200233, P.R. China

E-mail: junyang001fh@hotmail.com

Key words: intestinal microbiota depletion, protein-protein interaction network, modules, pathway enrichment analysis neuropeptide signaling pathway is reported to be affected by intestinal microbiota depletion in mice. The present study provides a list of candidate genes and processes related to the interaction of microbiota with the intestinal tract.

\section{Introduction}

There are $>1,000$ species of bacteria in the intestinal tract, known as intestinal microbiota. The genomes of these species encode $>100$-fold unique genes compared to the human genome (1). The intestinal microbiota is dominated by five bacterial phyla (Firmicutes, Bacteroidetes, Actinobacteria, Proteobacteria and Verrucomicrobia) and one Archaea (Euryarchaeota) (2). These complex communities of microorganisms play an important role in metabolic, nutritional, physiological and immunological processes in the human body (3). Molecular characterization of the intestinal microbiota by phylogenetic approaches has received considerable attention in recent years and revealed a remarkable compositional stability and resilience in adult life, even after pervasive treatments with antibiotics (4). Species of the genera Bifidobacterium and Lactobacillus are particularly present in the colon of healthy individuals, and they are generally regarded as desirable, owing to the reduction of the neutral $\mathrm{pH}$ to a more acidic $\mathrm{pH}$ that they cause (5). Changes in microbial community composition are closely associated with various diseases, such as allergic disease (6), colorectal cancer (7) and intestinal inflammatory disease (8).

Our understanding of intestinal microbiota and their importance for the human physiology has increased, owing to international research initiatives such as the MetaHIT project (1) and the Human Microbiome Project (9). However, the development of simple protocols for the manipulation of intestinal microbiota in experimental animal models is still needed. Recently, a study focusing on the effects of intestinal microbiota depletion on the gut mucosa and epithelial gene expression was performed; depletion of the intestinal microbiota was achieved in mice by administering broad-spectrum antibiotics in drinking water (10). The study reported that antibiotic treatment significantly reduced the expression of antimicrobial factors to a level similar to that of germ-free mice, and altered the expression of a total of 517 genes in the colonic epithelium. The expression of genes involved in the 
cell cycle was significantly altered, concomitant with reduced epithelial proliferative activity in situ, as assessed by Ki-67 expression, which suggested that commensal microbiota drives cellular proliferation in the colonic epithelium (10). Metabolites produced by the gut microbiota community from processes such as oxidation reduction and lipid metabolism have been reported to considerably affect intestinal functions (1).

The present study used a previously released microarray dataset (10) to assess the effects of intestinal microbiota depletion in mice, by focusing on the gene expression profiles of colonic intestinal epithelial cells in the presence and absence of intestinal microbiota. These profiles were analyzed using a series of bioinformatic methods, including protein-protein interaction (PPI) network construction, module functional annotation and pathway enrichment analyses. Further research on the mechanisms identified here as affected by the intestinal microbiota depletion is planned for a future study.

\section{Materials and methods}

Affymetrix microarray analysis. The raw data and the probe annotation files from the gene expression profiling dataset GSE22648 (10; accession no. GDS3921) were downloaded from the Gene Expression Omnibus database (the National Center of Biotechnology Information; http://www.ncbi.nlm. nih.gov/geo/query/acc.cgi?acc=GSE22648). These data were obtained on a GPL6887 platform using MouseWG-6 v2.0 expression beadchips (Illumina, Inc., San Diego, CA, USA). Data from a total of 11 chips were analyzed, corresponding to colonic intestinal epithelial cell gene expression profiles of 5 replicates from mice with depleted intestinal microbiota and 6 replicates from control mice that were not treated with antibiotics (germ-free).

Identification and clustering analysis of differentially expressed genes (DEGs). The raw data were preprocessed using the Affy package in R (11). Differential expression analysis between the 5 intestinal microbiota-depleted and the 6 control samples was performed using limma, a linear regression model software package available in R (12), and multiple testing correction was performed using a Bayesian method (13). The DEGs between intestinal microbiotadepleted and control samples were defined as these genes showing a $\mid \log$ fold change (FC) $\mid>1$ and a false discovery rate $(\mathrm{FDR})<0.05$. To visualize the expression profiles of DEGs and all genes, unsupervised hierarchical clustering analysis was performed (14).

PPI network construction. The search tool for the retrieval of interacting genes (STRING) (15) database was used to retrieve the predicted interactions for the identified DEGs; the version 9.0 of STRING covers $>1,100$ completely sequenced species. All associations available in STRING are provided with a probabilistic confidence score, which was derived by separately benchmarking groups of associations against the a manually curated functional classification scheme (15). Each score represents a rough estimate of how likely a given association describes a functional linkage between two gene products. The DEGs with a confidence score $>0.8$ were selected to construct the PPI network, using the open-source Cytoscape software (16). Cytoscape (http://cytoscape.org/) allows visualizing complex networks and integrating these networks to any type of attribute data.

Functional analysis of modules from the PPI network. The MCODE (17) plugin in Cytoscape was used to further divide the PPI into modules, using a cutoff value for the connectivity degree of nodes (proteins in the network) $>2$. Gene Ontology (GO) functional annotation and enrichment analysis of genes in the resulting modules was performed using the PiNGO plugin in Cytoscape (18) with a threshold $\mathrm{P}<0.05$ based on a hypergeometric test.

Pathway analysis. Information on the biological pathways in which the module-related DEGs are involved was retrieved from the Kyoto Encyclopedia of Genes and Genomes pathways database (http://www.genome.jp/kegg/pathway.html) (19,20). Visualization of these pathways and enrichment analysis was performed using the GenMAPP software (19). GenMAPP is a powerful tool for graphically viewing microarray data in the context of pathway analysis in an intuitive manner for biologists, and it was previously used in the analysis of microarray data related to allergic disease (21). $\mathrm{P}<0.05$ was set as the threshold used for enrichment analysis of KEGG pathways.

\section{Results}

Identification of DEGs. The normalized expression values following preprocessing of the raw data are shown in Fig. 1. Differential expression analysis on these values using $\mathrm{FDR}<0.05$ and $\log \mathrm{FCl}>1$ as cutoff criteria identified a total of 53 genes differentially expressed between depleted intestinal microbiota and control mice. Among these DEGs, 26 were upregulated and 27 were downregulated upon microbiota depletion.

Clustering analysis of DEGs. Hierarchical clustering analysis was performed on the expression values of all genes and of the 53 DEGs. Clearly distinct expression patterns were observed between the microbiota-depleted and the control mice in both the total gene and DEG clustering analysis (Fig. 2).

PPI analysis and module functional annotation. The PPI network was constructed (Fig. 3A) based on the predicted interactions of 14 DEGs showing a confidence score $>0.8$. Using the MCODE plugin in Cytoscape, the PPI network was divided into three modules (Fig. 3B-D). Modules 1, 2 and 3 were found to be significantly enriched $(\mathrm{P}<0.05)$ for 12, 14 and 14 Gene Ontology (GO) terms, respectively (Table I). The two most significant GO terms in module 1 were oxidation reduction $(\mathrm{P}=1.9321 \mathrm{E}-21)$ and metabolic process $(\mathrm{P}=1.1226 \mathrm{E}-12)$. The DEGs in module 1 (Fig. 3B), i.e., the cytochrome P450 (CYP) 4B1 isozyme gene (CYP4B1), $C Y P 2 D 10$ and $C Y P 2 D 26$, which were upregulated (Table II), and $C Y P 2 C 55$, which was downregulated, were all involved in these two processes (Table I). In addition, CYP4B1 was found to be involved in all enriched GO term functions of module 1 . The terms unsaturated fatty acid, lipid, cellular lipid and fatty acid metabolic process were the most significantly 


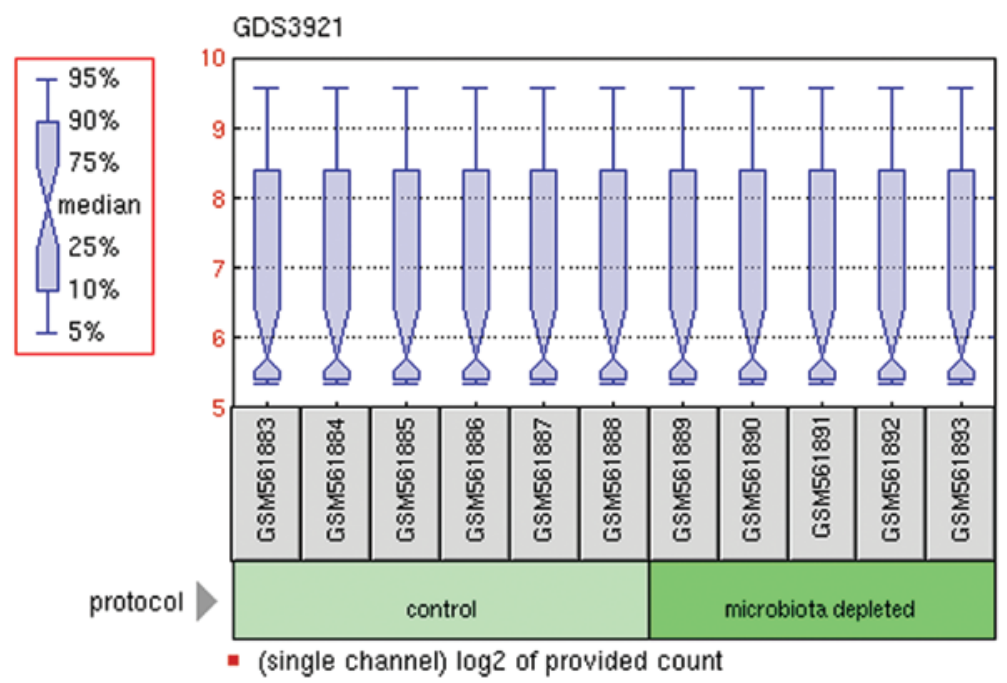

Figure 1. Boxplot of normalized expression values for the dataset (accession no. GDS3921). The data for the six control samples are presented on the left, and for the five samples with microbiota depletion on the right. The dotted line in the middle of each box represents the median of each sample, and its distribution among samples indicates the level of normalization of the data, with a nearly straight line revealing a fair normalization level.
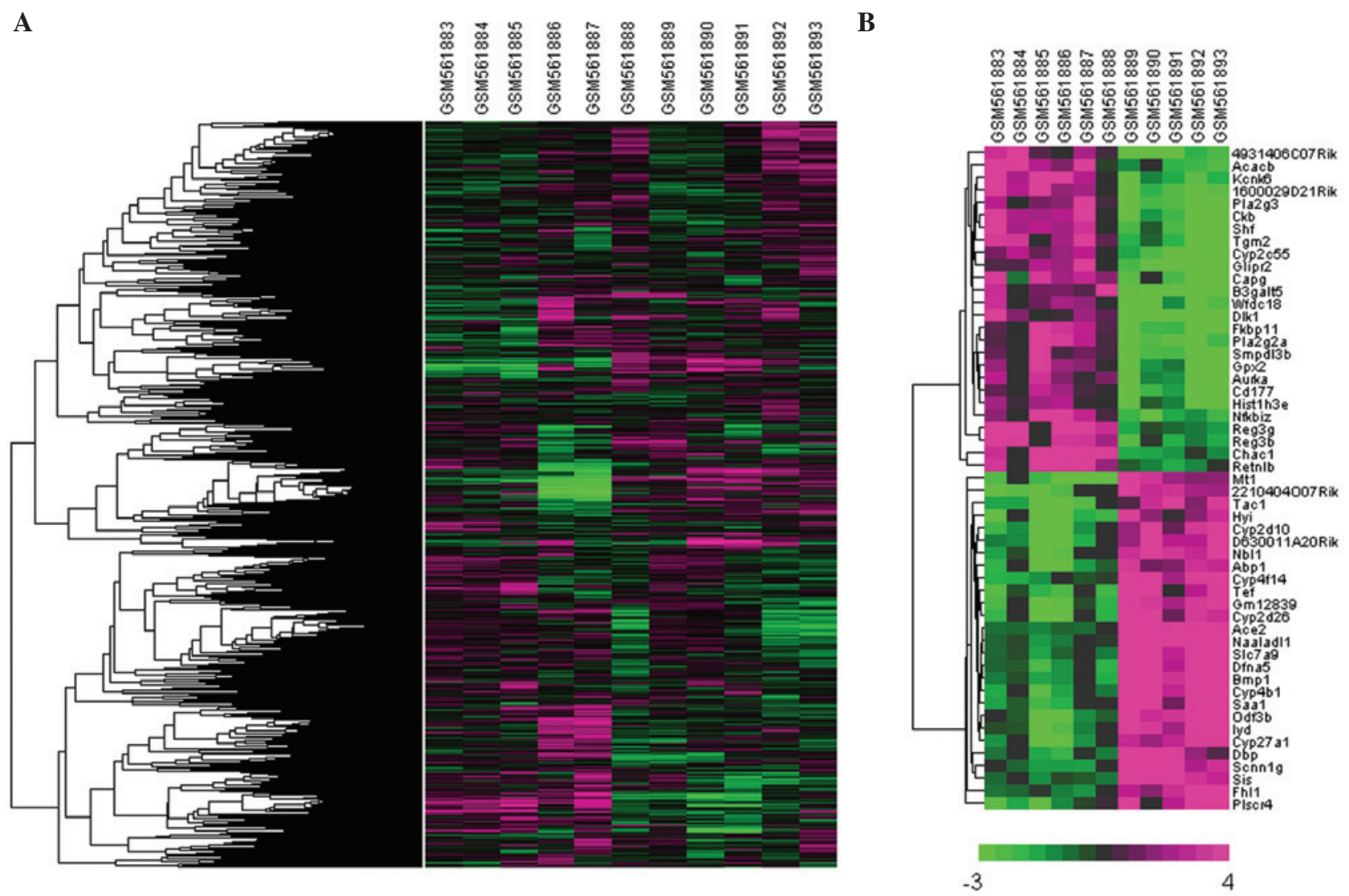

Figure 2. Clustering analysis of gene expression values of (A) all genes and (B) of differentially expressed genes. The change of color from green to red represents the change in $\operatorname{llog} \mathrm{FCl}$ from low to high. $\mathrm{FC}$, fold change.

enriched functions in module 2, and the upregulated gene CYP4F14 (Fig. 3C, Table II) was predicted to be involved in all these functions (Table I). Notably, the neuropeptide signaling pathway was the most significantly enriched function $(\mathrm{P}=2.5213 \mathrm{E}-11)$ in module 3 , and the upregulated gene tachykinin precursor 1 (TACl) (Fig. 3D, Table II) was predicted to be involved in this function (Table I). The top 5
DEGs in terms of significance (Table II) were all upregulated upon microbiota depletion.

Pathway analysis. Pathway enrichment analysis using the GenMAPP software was performed on the list of DEGs the products of which are parts of the three PPI modules. Module 1 was found to be significantly enriched for a total of 
Table I. Functional annotation of the genes in the three modules using Gene Ontology (GO) terms.

A, Significantly enriched GO terms $(n=12)$ and associated DEGs in module 1

\begin{tabular}{|c|c|c|c|}
\hline GO id & Corr. P & Genes in test set & Functional description \\
\hline 55114 & $1.9321 \mathrm{E}-21$ & $\begin{array}{l}C Y P 2 J 5, C Y P 2 C 70, C Y P 2 D 9, C Y P 2 D 10, C Y P 2 C 37, \\
C Y P 2 C 55, C Y P 3 A 25, C Y P 2 C 54, C Y P 3 A 13, C Y P 2 C 44, \\
C Y P 3 A 11, C Y P 2 C 29, C Y P 2 C 40, C Y P 2 E 1, C Y P 1 A 2, \\
C Y P 4 B 1, C Y P 2 D 26\end{array}$ & Oxidation reduction \\
\hline 8152 & $1.1226 \mathrm{E}-12$ & $\begin{array}{l}C Y P 2 J 5, C Y P 2 C 70, C Y P 2 D 9, C Y P 2 D 10, C Y P 2 C 37, \\
C Y P 2 C 55, C Y P 3 A 25, C Y P 2 C 54, C Y P 3 A 13, C Y P 2 C 44, \\
C Y P 3 A 11, C Y P 2 C 29, U G T 2 B 1, C Y P 2 C 40, C Y P 2 E 1, \\
C Y P 1 A 2, U G T 1 A 1, U G T 3 A 2, C Y P 4 B 1, C Y P 2 A 12, \\
\text { UGT2B35, UGT2B5, CYP2D26 }\end{array}$ & Metabolic process \\
\hline 42537 & $9.6276 \mathrm{E}-10$ & UGT2B1, CYP1A2, UGT1A1, CYP4B1 & Benzene and derivative metabolic process \\
\hline 6805 & 2.6493E-08 & $U G T 2 B 1, C Y P 1 A 2, U G T 1 A 1, C Y P 4 B 1$ & Xenobiotic metabolic process \\
\hline 71466 & 2.6493E-08 & $U G T 2 B 1, C Y P 1 A 2, U G T 1 A 1, C Y P 4 B 1$ & Cellular response to xenobiotic stimulus \\
\hline 6725 & $2.6493 \mathrm{E}-08$ & $\begin{array}{l}\text { CYP2A12, UGT2B35, UGT2B1, CYP1A2, } \\
\text { UGT1A1, CYP4B1 }\end{array}$ & $\begin{array}{l}\text { Cellular aromatic compound metabolic } \\
\text { process }\end{array}$ \\
\hline 9410 & $3.7437 \mathrm{E}-08$ & $U G T 2 B 1, C Y P 1 A 2, U G T 1 A 1, C Y P 4 B 1$ & Response to xenobiotic stimulus \\
\hline 70887 & $4.26 \mathrm{E}-04$ & $U G T 2 B 1, C Y P 1 A 2, U G T 1 A 1, C Y P 4 B 1$ & Cellular response to chemical stimulus \\
\hline 44248 & $1.01 \mathrm{E}-03$ & UGT2B35, UGT2B1, CYP1A2, UGT1A1, CYP4B1 & Cellular catabolic process \\
\hline 9056 & $3.27 \mathrm{E}-03$ & UGT2B35, UGT2B1, CYP1A2, UGT1A1, CYP4B1 & Catabolic process \\
\hline 42221 & $7.25 \mathrm{E}-03$ & UGT2B1, CYP2D26, CYP1A2, UGT1A1, CYP4B1 & Response to chemical stimulus \\
\hline 51716 & $9.66 \mathrm{E}-03$ & UGT2B1, CYP1A2, UGT1A1, CYP4B1 & Cellular response to stimulus \\
\hline
\end{tabular}

B, Significantly enriched GO terms $(n=14)$ and associated genes in module 2

\begin{tabular}{|c|c|c|c|}
\hline GO id & Corr. P & Genes in test set & Functional description \\
\hline 33559 & $2.0285 \mathrm{E}-08$ & PTGS2, PTGS1, CYP4F14, ALOX5 & Unsaturated fatty acid metabolic process \\
\hline 6629 & $1.1496 \mathrm{E}-07$ & PTGS2, PLB1, PTGS1, PLA2G2A, CYP4F14, ALOX5 & Lipid metabolic process \\
\hline 44255 & $1.2555 \mathrm{E}-06$ & PTGS2, PTGS1, PLA2G2A, CYP4F14, ALOX5 & Cellular lipid metabolic process \\
\hline 6631 & $2.1203 \mathrm{E}-06$ & PTGS2, PTGS1, CYP4F14, ALOX5 & Fatty acid metabolic process \\
\hline 32787 & $9.1722 \mathrm{E}-06$ & PTGS2, PTGS1, CYP4F14, ALOX5 & Monocarboxylic acid metabolic process \\
\hline 43436 & $5.05 \mathrm{E}-05$ & PTGS2, PTGS1, CYP4F14, ALOX5 & Oxoacid metabolic process \\
\hline 19752 & $5.05 \mathrm{E}-05$ & PTGS2, PTGS1, CYP4F14, ALOX5 & Carboxylic acid metabolic process \\
\hline 6082 & $5.05 \mathrm{E}-05$ & PTGS2, PTGS1, CYP4F14, ALOX5 & Organic acid metabolic process \\
\hline 42180 & $5.26 \mathrm{E}-05$ & PTGS2, PTGS1, CYP4F14, ALOX5 & Cellular ketone metabolic process \\
\hline 55114 & $8.20 \mathrm{E}-05$ & PTGS2, PTGS1, CYP4F14, ALOX5 & Oxidation reduction \\
\hline 44281 & $1.02 \mathrm{E}-03$ & PTGS2, PTGS1, CYP4F14, ALOX5 & Small molecule metabolic process \\
\hline 44238 & $1.89 \mathrm{E}-03$ & PTGS2, PLB1, PTGS1, PLA2G2A, CYP4F14, ALOX5 & Primary metabolic process \\
\hline 8152 & $3.03 \mathrm{E}-03$ & PTGS2, PLB1, PTGS1, PLA2G2A, CYP4F14, ALOX5 & Metabolic process \\
\hline 44237 & $9.30 \mathrm{E}-03$ & PTGS2, PTGS1, PLA2G2A, CYP4F14, ALOX5 & Cellular metabolic process \\
\hline
\end{tabular}

C, Significantly enriched GO terms $(n=14)$ and associated genes in module 3

\begin{tabular}{llll}
\hline GO id & Corr. P & Genes in test set & Functional description \\
\hline 7218 & $2.5213 \mathrm{E}-11$ & GRP, TACR3, TACR2, TACR1, TAC1, TAC2 & Neuropeptide signaling pathway \\
8015 & $1.8064 \mathrm{E}-10$ & NTS, TACR3, TACR1, AGT, TAC1, TAC2 & Blood circulation
\end{tabular}


Table I. Continued.

\begin{tabular}{|c|c|c|c|}
\hline GO id & Corr. P & Genes in test set & Functional description \\
\hline 3013 & $1.8064 \mathrm{E}-10$ & NTS, TACR3, TACR1, AGT, TAC1, TAC2 & Circulatory system process \\
\hline 7186 & $1.1712 \mathrm{E}-07$ & GRP, TACR3, TACR2, TACR1, AGT, TAC1, GAST, TAC2 & $\begin{array}{l}\text { G-protein coupled receptor } \\
\text { protein signaling pathway }\end{array}$ \\
\hline 7166 & $8.8661 \mathrm{E}-07$ & GRP, TACR3, TACR2, TACR1, AGT, TAC1, GAST, TAC2 & $\begin{array}{l}\text { Cell surface receptor linked } \\
\text { signaling pathway }\end{array}$ \\
\hline 23033 & 4.6021E-06 & GRP, TACR3, TACR2, TACR1, AGT, TAC1, GAST, TAC2 & Signaling pathway \\
\hline 51239 & $4.8121 \mathrm{E}-06$ & GRP, TACR3, TACR2, TACR1, AGT, TAC1 & $\begin{array}{l}\text { Regulation of multicellular } \\
\text { organismal process }\end{array}$ \\
\hline 3008 & 4.9678E-06 & NTS, TACR3, TACR2, TACR1, AGT, TAC1, TAC2 & System process \\
\hline 65008 & $1.24 \mathrm{E}-05$ & NTS, TACR3, TACR1, AGT, TAC1, TAC2 & Regulation of biological quality \\
\hline 23052 & $1.33 \mathrm{E}-05$ & GRP, TACR3, TACR2, TACR1, AGT, TAC1, GAST, TAC2 & Signaling \\
\hline 65007 & $4.55 \mathrm{E}-05$ & GRP, NTS, TACR3, TACR2, TACR1, AGT, TAC1, GAST, TAC2 & Biological regulation \\
\hline 32501 & 4.32E-04 & NTS, TACR3, TACR2, TACR1, AGT, TAC1, TAC2 & Multicellular organismal process \\
\hline 50794 & $2.08 \mathrm{E}-03$ & GRP, TACR3, TACR2, TACR1, AGT, TAC1, GAST & Regulation of cellular process \\
\hline 50789 & 2.69E-03 & GRP, TACR3, TACR2, TACR1, AGT, TAC1, GAST & Regulation of biological process \\
\hline
\end{tabular}

Corr. P, corrected p-value; $C Y P$, cytochrome $\mathrm{P} 450$ gene; TAC1, tachykinin precursor 1 gene.

Table II. Characteristics of the most significant differentially expressed genes in the 3 modules.

\begin{tabular}{|c|c|c|c|c|c|}
\hline Id & Gene symbol & FDR & $\log \mathrm{FCl}$ & GO terms ${ }^{\mathrm{a}}$ & Regulation \\
\hline ILMN_2790496 & $C Y P 4 B 1$ & 0.0023580 & 2.64 & All in Table IA & Up \\
\hline ILMN_1229535 & $C Y P 2 D 10$ & 0.0234023 & 1.42 & 55114,8152 & Up \\
\hline ILMN_2704777 & $C Y P 2 D 26$ & 0.0190232 & 1.92 & $55114,8152,42221$ & Up \\
\hline ILMN_1231625 & CYP4F 14 & 0.0055720 & 1.31 & All in Table IB & Up \\
\hline ILMN_1251000 & TAC1 & 0.0257095 & 1.09 & All in Table IC & Up \\
\hline
\end{tabular}

${ }^{a}$ The numbers denote Gene Ontology (GO) ids shown in Table I. FC, fold change; CYP, cytochrome P450; TAC1, tachykinin precursor 1; FDR, false discovery rate.

12 pathways, module 2 for 5 and module 3 for 2 (Table III). The most significant pathways in module 1 included metabolism of xenobiotics by $\mathrm{P} 450 \mathrm{~s}(\mathrm{P}=2.20 \mathrm{E}-26)$, linoleic acid metabolism $(\mathrm{P}=6.54 \mathrm{E}-24)$ and arachidonic acid metabolism $(\mathrm{P}=1.49 \mathrm{E}-10)$. Significant pathways in module 2 also included arachidonic acid metabolism $(\mathrm{P}=5.61 \mathrm{E}-10)$ and linoleic acid metabolism $(\mathrm{P}=3.95 \mathrm{E}-02)$. Two pathways were significantly enriched in module 3 , the calcium signaling pathway $(\mathrm{P}=3.23 \mathrm{E}-03)$ and neuroactive ligand-receptor interaction $(\mathrm{P}=5.95 \mathrm{E}-03)$.

\section{Discussion}

The collective genome of the human intestinal microbiota was estimated to contain 3.3 million microbial genes, which is $~ 150$ times more genes than the human genome. Intestinal microbiota mostly use fermentation to generate energy, converting sugars, in part, to short-chain fatty acids, which are used by the host as an energy source (1). To understand the impact of intestinal microbiota on human health, it is crucial to assess their potential function. The present study identified a total of 53 DEGs, comprising 26 upregulated and 27 downregulated genes upon depletion of the intestinal microbiota in mice. Important differences in gene expression were observed between intestinal microbiota-depleted and control mice in hierarchical clustering analysis. The PPI network of DEGs was constructed and divided into 3 modules, with the most significant module-related DEGs being CYP4B1 in module 1, CYP4F14 in module 2 and TAC1 in module 3 . The majority of enriched pathways of module 1 and 2 were oxidation reduction (metabolism of xenobiotics by CYPs) and lipid (e.g., linoleic and arachidonic acid) metabolism pathways. In addition, the neuropeptide signaling pathway was the most significantly enriched pathway in module 3 .

Two types of functions of intestinal microbiota have been identified in a previous study, those required in all bacteria and those potentially specific to the gut (1). Functions of 
A

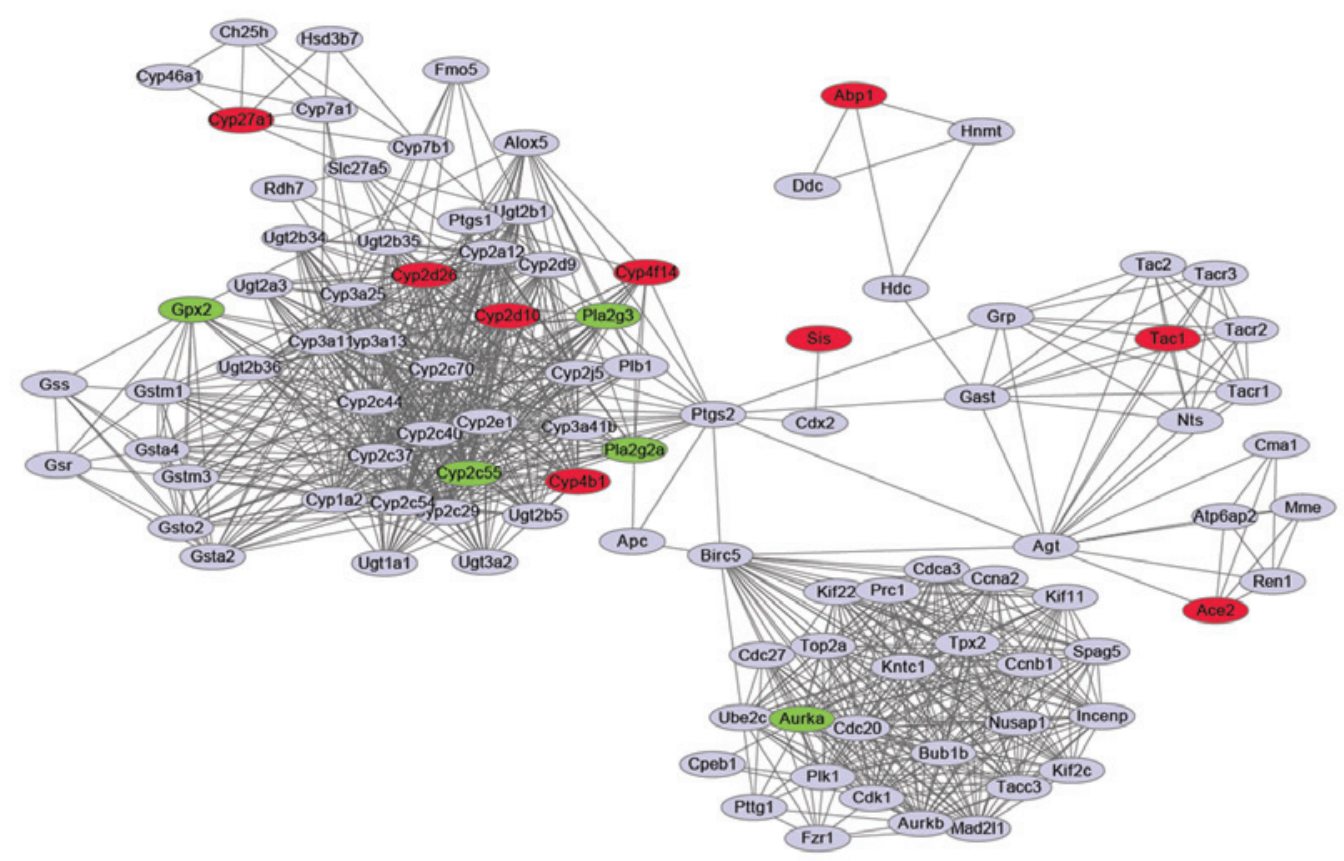

B

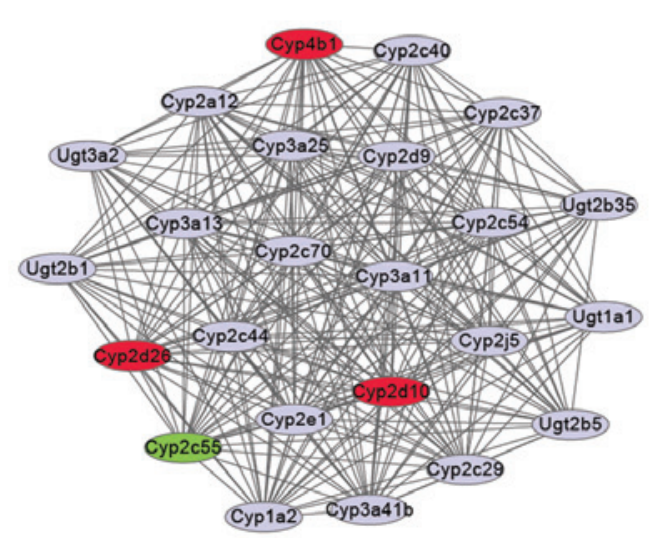

C

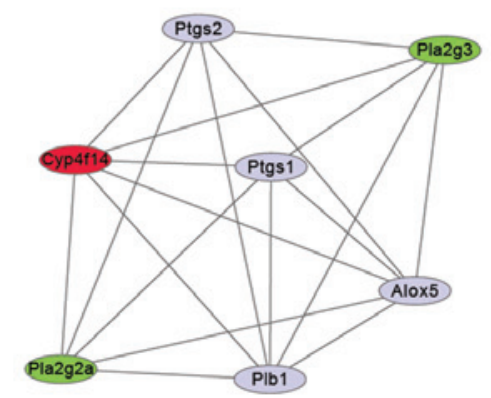

D

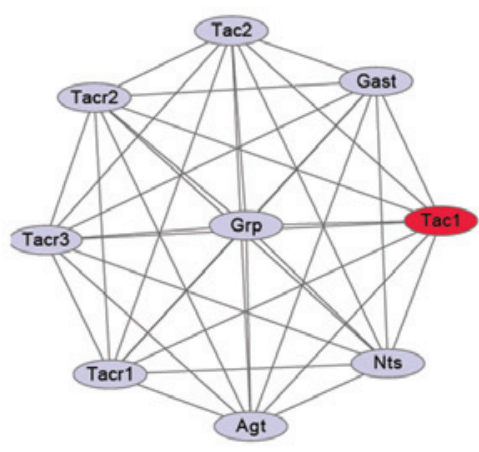

Figure 3. Primary protein-protein interaction (PPI) network and selected modules. (A) PPI network for products of differentially expressed genes (DEGs). (B-D) Modules including significant DEGs (confidence score $>0.8$ ). Red- and green-color nodes represent products of up- and downregulated DEGs, respectively. Purple nodes denote products of genes predicted to interact with the DEGs.

the first category relate to central metabolic pathways (for example, carbon metabolism and amino acid synthesis) and to important protein complexes (RNA and DNA polymerase, ATP synthase, general secretory apparatus) (1). The putative gut-specific functions include those involved in adhesion to host proteins (collagen, fibrinogen, fibronectin), or in harvesting sugars of the globo-series glycolipids, which are carried on blood and epithelial cells (1). In the present study, most of module 1-related DEGs were involved in oxidation reduction and metabolic processes such as metabolism of xenobiotics by CYPs, and the majority of module 2-related DEGs were involved in lipid metabolic processes, such as lipid metabolic process and arachidonic acid metabolism. These results suggest that the intestinal microbiota is involved in numerous metabolic and biosynthetic processes, but has particularly important roles in the regulation of lipid biosynthesis and in oxidation-reduction processes, as also indicated by previous studies (22-24).
Further analysis of the most significant DEGs $C Y P 4 B 1$, CYP2D10, CYP2D26 (module 1) and CYP4F14 (module 2) revealed that $C Y P 4 B 1$ and $C Y P 4 F 14$ are involved in almost all of the functions of each PPI module. In rats and rabbits, the CYP4B1 protein was shown to play an important role in mutagenic activation of procarcinogens in the organs (25). Most of organic xenobiotics require metabolic activation to electrophilic intermediates to produce adverse carcinogenic effects. Specific enzymes of the CYP superfamily are involved in the formation of reactive metabolites from certain substrates that are predicted or known occupational and environmental carcinogens (26). A new prodrug-activating enzyme system for pharmacogenic therapy of experimental brain tumors based on the rabbit CYP4B1 protein was previously described (27). CYP4Fs are a subfamily of enzymes involved in arachidonic acid metabolism and showing the highest catalytic activity towards leukotriene (LT)B4, a potent chemoattractant involved in inflammation. CYP4F-mediated metabolism of LTB4 leads 
Table III. Pathway enrichment analysis of differentially expressed genes in the three modules based on information from the Kyoto Encyclopedia of Genes and Genomes (KEGG) pathways database for Mus musculus (mmu).

\begin{tabular}{|c|c|c|}
\hline Module & KEGG id & $\mathrm{P}$ \\
\hline \multirow[t]{12}{*}{1} & mmu00980: Metabolism of xenobiotics by cytochrome P450 & $2.20 \mathrm{E}-26$ \\
\hline & mmu00830: Retinol metabolism & $3.63 \mathrm{E}-26$ \\
\hline & mmu00591: Linoleic acid metabolism & $6.54 \mathrm{E}-24$ \\
\hline & mmu00983: Drug metabolism & $1.30 \mathrm{E}-10$ \\
\hline & mmu00590: Arachidonic acid metabolism & $1.49 \mathrm{E}-10$ \\
\hline & mmu00140: Steroid hormone biosynthesis & $5.88 \mathrm{E}-09$ \\
\hline & mmu00053: Ascorbate and aldarate metabolism & $1.18 \mathrm{E}-03$ \\
\hline & mmu00040: Pentose and glucuronate interconversions & $1.52 \mathrm{E}-03$ \\
\hline & mmu00860: Porphyrin and chlorophyll metabolism & 4.74E-03 \\
\hline & mmu00150: Androgen and estrogen metabolism & $4.74 \mathrm{E}-03$ \\
\hline & mmu00500: Starch and sucrose metabolism & $6.77 \mathrm{E}-03$ \\
\hline & mmu00232: Caffeine metabolism & $3.10 \mathrm{E}-02$ \\
\hline \multirow[t]{5}{*}{2} & mmu00590: Arachidonic acid metabolism & $5.61 \mathrm{E}-10$ \\
\hline & mmu04370: Vascular endothelial growth factor signaling pathway & $1.69 \mathrm{E}-03$ \\
\hline & mmu00592: $\alpha$-linolenic acid metabolism & $1.56 \mathrm{E}-02$ \\
\hline & mmu00565: Ether lipid metabolism & $3.01 \mathrm{E}-02$ \\
\hline & mmu00591: Linoleic acid metabolism & $3.95 \mathrm{E}-02$ \\
\hline \multirow[t]{2}{*}{3} & mmu04020: Calcium signaling pathway & $3.23 \mathrm{E}-03$ \\
\hline & mmu04080: Neuroactive ligand-receptor interaction & $5.95 \mathrm{E}-03$ \\
\hline
\end{tabular}

to inactive $\omega$-hydroxy products, incapable of initiating chemotaxis and the inflammatory stimuli that result in the influx of inflammatory cells (28). The CYP4B1 and CYP4F14 genes were identified as significantly upregulated in the present study, which, in combination with previous reports, suggests that intestinal microbiota depletion may lead to inflammation and cancer in the body.

It is notable that modules 1 and 2 were both enriched for the processes of arachidonic and linoleic acid metabolism. Arachidonic acid is a polyunsaturated $\omega-6$ fatty acid that is released in response to tissue injury. Arachidonic acid is a pivotal signaling molecule, involved in the initiation and propagation of diverse signaling cascades regulating inflammation, pain and homeostatic functions (29). It is metabolized by three enzymatic pathways: the cyclooxygenase pathway produces prostanoid, the lipoxygenase pathway yields monohydroxy compounds and LTs, while the CYP epoxygenase pathway generates hydroxy and epoxyeicosanoids. There is increasing evidence that some of these metabolic products play critical roles in cardiovascular disease (29). Linoleic acid is predominant in dairy products and plant oils such as flax seed, and animal studies have reported a reduction in intra-abdominal fat and an enhanced gain in fat-free mass upon linoleic acid supplementation; another study reported linoleic acid-mediated whole-body fat loss in overweight men and women; there have also been some concerns that linoleic acid can promote oxidative stress and induce hepatic lipid accumulation (30-32). Based on these studies and the present findings on arachidonic and linoleic acid metabolism, the two processes appear to play a key role in human health and to be closely linked to the balance of intestinal microbiota.

In contrast to the reported effects of intestinal microbiota on oxidation reduction and lipid metabolism (30-32), an association between intestinal microbiota and the neuropeptide signaling pathway has not been previously reported. In our study, it is notable that the neuropeptide signaling pathway was the most significantly enriched pathway in module 3 . Among the here-identified DEGs, TAC1 is predicted to be involved in this pathway. This gene encodes a neurotransmitter of the central and peripheral nervous system (33), and the protein has additionally been associated with immunologic and inflammatory processes (34). The gut and the brain are closely connected organs, and their interaction plays an important role not only in gastrointestinal function, but also in certain feeling states and in intuitive decision making (35); alterations in this interaction have been associated with a wide range of disorders, including functional, inflammatory gastrointestinal, and eating disorders. It has been reported that healthy humans and rats produce autoantibodies directed against appetite-regulating peptide hormones and neuropeptides, suggesting that these autoantibodies may play physiological roles in hunger- and satiety-related pathways (36). Gut-related antigens including those produced by the intestinal microflora, may affect the production of these autoantibodies, which might represent a new link between the gut and the regulation of appetite. We thus argue that 
the depletion of the intestinal microflora in mice may lead to impaired neuropeptide signaling.

In conclusion, our findings strongly suggest that intestinal microbiota depletion affects metabolism, oxidation reduction and neuropeptide signaling pathways in mice, involving a number of genes and interactions. Numerous diseases, as well as aging, can be induced by depletion of the intestinal microflora, and therefore, the dynamic equilibrium of the intestinal microflora plays a key role in human health. The neuropeptide signaling pathway was first reported in the present study to be affected by the depletion of the intestinal microflora, a result which reveals a potential link between the intestinal bacteria and the nervous system. However, further experimentation and additional studies are needed to confirm this link; such studies are expected to enhance our understanding of the interactions between the bacteria of the intestinal microflora and their host environment.

\section{References}

1. Qin J, Li R, Raes J, et al: A human gut microbial gene catalogue established by metagenomic sequencing. Nature 464: 59-65, 2010.

2. Tremaroli V and Backhed F: Functional interactions between the gut microbiota and host metabolism. Nature 489: 242-249, 2012.

3. Gerritsen J, Smidt H, Rijkers GT and de Vos WM: Intestinal microbiota in human health and disease: the impact of probiotics. Genes Nutr 6: 209-240, 2011.

4. Jernberg C, Lofmark S, Edlund C and Jansson JK: Long-term impacts of antibiotic exposure on the human intestinal microbiota. Microbiology 156: 3216-3223, 2010.

5. Arroyo G, Penas E, Pedrazuela A and Prestamo G: Intestinal microbiota in rats fed with tofu (soy curd) treated under high pressure. Eur Food Res Technol 220: 395-400, 2005.

6. Bjorksten B, Sepp E, Julge K, Voor T and Mikelsaar M: Allergy development and the intestinal microflora during the first year of life. J Allergy Clin Immunol 108: 516-520, 2001.

7. Ohigashi S, Sudo K, Kobayashi D, et al: Changes of the intestinal microbiota, short chain fatty acids, and fecal $\mathrm{pH}$ in patients with colorectal cancer. Dig Dis Sci 58: 1717-1726, 2013.

8. Rawls JF: Enteric infection and inflammation alter gut microbial ecology. Cell Host Microbe 16: 73-74, 2007.

9. Human Microbiome Jumpstart Reference Strains Consortium, Nelson KE, Weinstock GM, Highlander SK, et al: A catalog of reference genomes from the human microbiome. Science 328: 994-999, 2010

10. Reikvam DH, Erofeev A, Sandvik A, et al: Depletion of murine intestinal microbiota: effects on gut mucosa and epithelial gene expression. PLoS One 6: e17996, 2011.

11. R. Irizarry, L. Gautier, and L. Cope. An R package for analyses of Affymetrix oligonucleotide arrays. In: The Analysis of Gene Expression Data: Methods and Software. G. Parmigiani, E. S. Garrett, R. A. Irizarry, and S. L. Zeger (eds). Springer-Verlag, Berlin, pp102-119, 2003.

12. Smyth GK: limma: linear models for microarray data. In: Bioinformatics and Computational Biology Solutions Using R and Bioconductor. Gentleman R, Carey VJ, Huber W, Irizarry RA and Dudoit S (eds). Springer New York, NY, pp397-420, 2005.

13. Shao X, Huang B, Lee JM, Xu F and Espejo A: Bayesian method for multirate data synthesis and model calibration. AIChE J 57: 1514-1525, 2011.

14. Hur AB, Elisseeff A and Guyon I: Identification of co-regulation patterns by unsupervised cluster analysis of gene expression data. US Patent 8,489,531. Filed February 2, 2011; issued July 16, 2013.
15. Szklarczyk D, Franceschini A, Kuhn M, et al: The STRING database in 2011: functional interaction networks of proteins, globally integrated and scored. Nucleic Acids Res 39: D561-D568, 2011.

16. Shannon P, Markiel A, Ozier O, et al: Cytoscape: a software environment for integrated models of biomolecular interaction networks. Genome Res 13: 2498-2504, 2003.

17. Bader GD and Hogue CW: An automated method for finding molecular complexes in large protein interaction networks. BMC Bioinformatics 4: 2, 2003.

18. Smoot M, Ono K, Ideker T and Maere S: PiNGO: a Cytoscape plugin to find candidate genes in biological networks. Bioinformatics 27: 1030-1031, 2011.

19. Kanehisa M and Goto S: KEGG: kyoto encyclopedia of genes and genomes. Nucleic Acids Res 28: 27-30, 2000.

20. Xue J, Zhao C and Liang A: Pathway analysis of the differential expression genes of oligonucleotide microarray of airway allergic diseases using GenMAPP. Lin Chung Er Bi Yan Hou Tou Jing Wai Ke Za Zhi 25: 371-373, 2011 (In Chinese).

21. Dahlquist KD, Salomonis N, Vranizan K, Lawlor SC and Conklin BR: GenMAPP, a new tool for viewing and analyzing microarray data on biological pathways. Nat Genet 31: 19-20, 2002.

22. Velagapudi VR, Hezaveh R, Reigstad CS, et al: The gut microbiota modulates host energy and lipid metabolism in mice. J Lipid Res 51: 1101-1112, 2010.

23. Bäckhed F: Programming of host metabolism by the gut microbiota. Ann Nutr Metab 58: 44-52, 2011.

24. Espey MG: Role of oxygen gradients in shaping redox relationships between the human intestine and its microbiota. Free Radic Biol Med 55: 130-140, 2013.

25. Tamaki Y, Arai T, Sugimura H, et al: Association between cancer risk and drug-metabolizing enzyme gene (CYP2A6, CYP2A13, CYP4B1, SULT1A1, GSTM1, and GSTT1) polymorphisms in cases of lung cancer in Japan. Drug Metab Pharmacokinet 26: 516-522, 2011.

26. Roos PH, Belik R, Follmann W, et al: Expression of cytochrome P450 enzymes CYP1A1, CYP1B1, CYP2E1 and CYP4B1 in cultured transitional cells from specimens of the human urinary tract and from urinary sediments. Arch Toxicol 80: 45-52, 2006.

27. Jang SJ, Kang JH, Lee TS, et al: Prodrug-activating gene therapy with rabbit cytochrome P450 4B1/4-ipomeanol or 2-aminoanthracene system in glioma cells. Nucl Med Mol Imaging 44: 193-198, 2010.

28. Antonovic L: A physiological role of cytochromes P450 4Fs: mouse model (unpublished dissertation). The University of Texas, AAI3218710, 2006.

29. Li N, Liu JY, Qiu H, et al: Use of metabolomic profiling in the study of arachidonic acid metabolism in cardiovascular disease. Congest Heart Fail 17: 42-46, 2011.

30. Gaullier JM, Halse J, Hoye K, et al: Supplementation with conjugated linoleic acid for 24 months is well tolerated by and reduces body fat mass in healthy, overweight humans. J Nutr 135: 778-784, 2005.

31. Gaullier JM, Halse J, Hoye K, et al: Conjugated linoleic acid supplementation for $1 \mathrm{y}$ reduces body fat mass in healthy overweight humans. Am J Clin Nutr 79: 1118-1125, 2004.

32. Kreider RB, Ferreira MP, Greenwood M, Wilson M and Almada AL: Effects of conjugated linoleic acid supplementation during resistance training on body composition, bone density, strength, and selected hematological markers. J Strength Cond Res 16: 325-334, 2002.

33. Graham GJ, Stevens JM, Page NM, et al: Tachykinins regulate the function of platelets. Blood 104: 1058-1065, 2004.

34. Brain SD: Sensory neuropeptides: their role in inflammation and wound healing. Immunopharmacology 37: 133-152, 1997.

35. Mayer EA: Gut feelings: the emerging biology of gut-brain communication. Nat Rev Neurosci 12: 453-466, 2011.

36. Vijay-Kumar M, Aitken JD, Carvalho FA, et al: Metabolic syndrome and altered gut microbiota in mice lacking Toll-like receptor 5. Science 328: 228-231, 2010. 